\title{
Primary aldosteronism associated with severe hypokalemic rhabdomyolysis
}

\author{
Wan-Ting Tsai, ${ }^{1,6}$ Yen-Lin Chen, ${ }^{7}$ Wei-Shiung Yang, ${ }^{1,2}$ Hong-Da Lin, ${ }^{3,7}$ \\ Chih-Cheng Chien, ${ }^{4}$ Ching-Ling Lin ${ }^{5}$
}

\begin{abstract}
${ }^{1}$ Graduate Institute of Clinical Medicine, College of Medicine, National Taiwan University; ${ }^{2}$ Division of Endocrinology and Metabolism, Department of Internal Medicine, National Taiwan University Hospital; ${ }^{3}$ Division of Endocrinology and Metabolism, Department of Internal Medicine, Taipei Veterans General Hospital; ${ }^{4}$ Department of Anesthesiology, Sijhih Cathay General Hospital; ${ }^{5}$ Division of Endocrinology and Metabolism, Department of Internal Medicine, Cathay general hospital; ' ${ }^{6}$ Division of Endocrinology and Metabolism, Department of Internal Medicine, Sijhih Cathay General Hospital; ' Division of Endocrinology and Metabolism, Department of Internal Medicine, Shin Kong Wu Ho-Su Memorial Hospital, Taiwan
\end{abstract}

Dear Sir,

Reports associating hypokalemic rhabdomyolysis with primary aldosteronism are rare.$^{1,2}$ Hypokalemia does not develop in every patient with primary aldosteronism, and hypokalemic rhabdomyolysis is even rarer in patients with primary aldosteronism. ${ }^{3} \mathrm{We}$ present here a patient with primary aldosteronism, who developed hypokalemia and rhabdomyolysis. This cases furnishes an instructive example of the potential for rhabdomyolysis to develop in patients with primary aldosteronism.

A 49-year-old hypertensive woman was sent to our emergency room for general weakness mainly involving her two legs. Her serum potassium was extremely low $(1.8 \mathrm{mEq} / \mathrm{L})$ and her creatine phosphokinase 1,753 $\mathrm{U} / \mathrm{L}$ (normal levels 90-140). Base on these data and the clinical picture hypokalemic rhabdomyolysis was

Address for correspondence:

Yen-Lin Chen, MD, Division of Endocrinology, Department of Internal Medicine, Shin Kong Wu Ho-Su Memorial Hospital, No. 95 Wen-Chang Road, Taipei 11120, Taiwan, Tel.: 886-2-2833-2211 ext. 2031, Fax: 886-2-2704-4109, e-mail: M004330@ms.skh.org.tw

Received 03-05-12, Revised 16-06-12, Accepted 01-07-12 diagnosed. She had a previous history of intracranial hemorrhage, primary aldosteronism, and hypertension, all diagnosed two years previously. At that time, low renin $(0.35 \mathrm{pg} / \mathrm{ml})$, high aldosterone $(336.73 \mathrm{pg} /$ $\mathrm{ml}$ ), and high aldosterone-renin ratio (ARR, 962) had been noted. Abdominal CT showed bilateral adrenal tumors, both homogenous, hypodense, right $2.29 \mathrm{~cm}$ and left $0.92 \mathrm{~cm}$ in diameter (Figure 1). She received right laparoscopic adrenalectomy and a $2 \times 2$ $\mathrm{cm}$ tumor was extirpated. Hypokalemia resolved after the operation but hypertension persisted, which was controlled by two anti-hypertensive agents, amlodipine and valsartan. However, hypokalemia $(1.8 \mathrm{mEq} / \mathrm{L}$, with CPK 1,753U/L), low renin $(1.06 \mathrm{pg} / \mathrm{ml})$, high aldosterone (648.9 pg/ml), and high ARR ( 677) were determined at this admission. The patient also had metabolic alkalosis, with positve electrocardiogram change ( $\mathrm{U}$ wave and inverted $\mathrm{T}$ wave). Abdominal CT showed a left adrenal tumor measuring $1.5 \mathrm{~cm}$ in diameter. After treatment with hydration, potassium replacement, and spironolactone, her rhabdomyolysis resolved in 10 days (CPK from 1,753 to $72 \mathrm{U} / \mathrm{L}$, Figure 2). Since the patient refused surgery for left side adrenal tumor, she continued receiving medical therapy: her blood pressure was around $130 / 85 \mathrm{mmHg}$ 

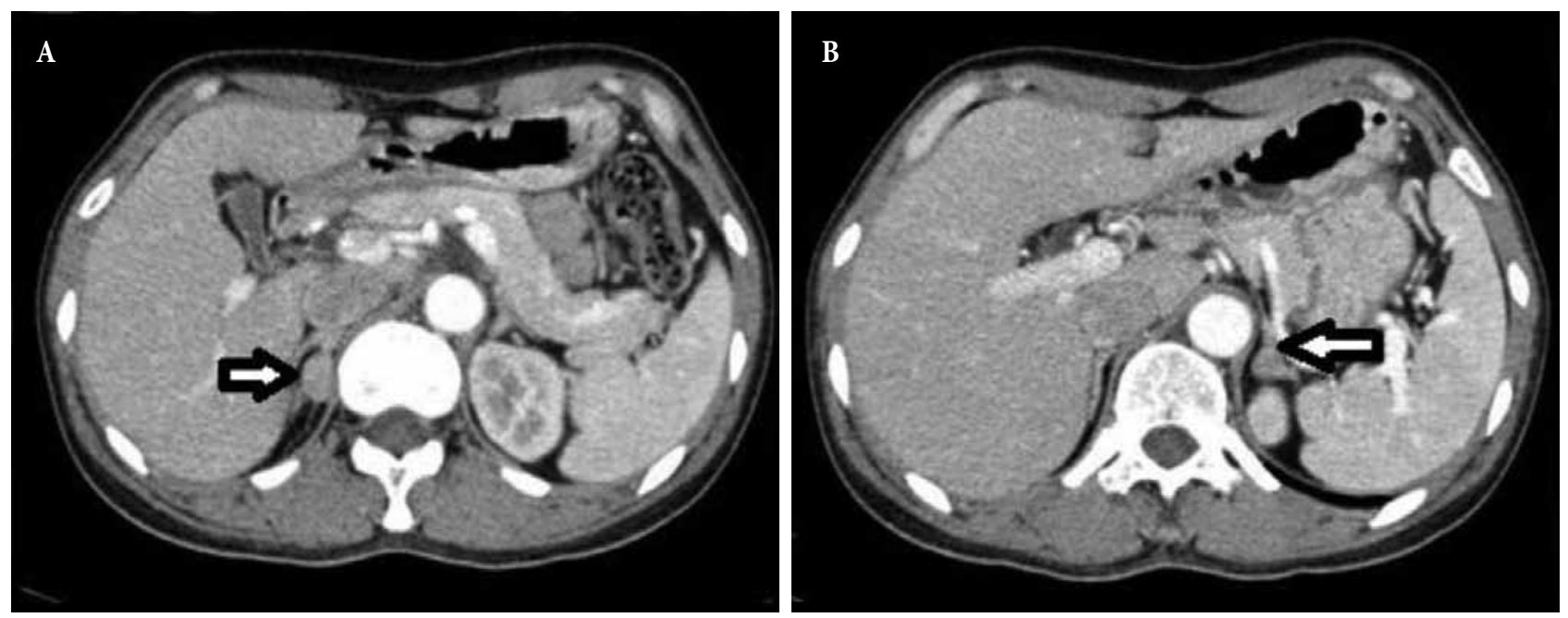

Figure 1. Adrenal CT with contrast medium showing bilateral adrenal tumors. (A) Right adrenal tumor measuring $2.29 \mathrm{~cm}$ in diameter (arrow). (B) Left adrenal tumor measuring $0.92 \mathrm{~cm}$ in diameter (arrow).

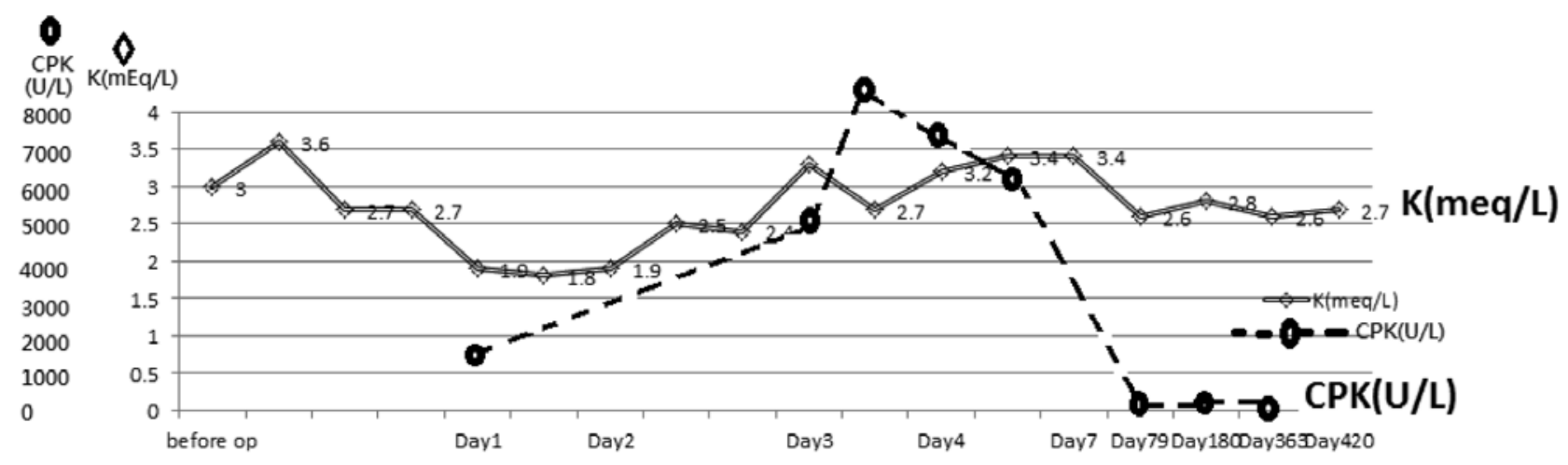

Figure 2. Serum CPK and potassium level two years after laparoscopic right adrenalectomy. (O: CPK level (U/L); $\diamond$ : potassium level $(\mathrm{mEq} / \mathrm{L})$.

and her potassium was normal.

Few cases have been reported of primary aldosteronism being related to hypokalemic rhabdomyolysis, and in these cases the potassium levels were all under $2.0 \mathrm{mEq} / \mathrm{L}$, except one $(2.1 \mathrm{mEq} / \mathrm{L})$ in Goto's report. ${ }^{3-5} \mathrm{We}$ concluded that patients with severe hypokalemia (potassium level below $2 \mathrm{mEq} / \mathrm{L}$ ) are at high risk for hypokalemic rhabdomyolysis. Mineralocorticoid antagonist is the standard medical treatment for patients with primary aldosteronism. Anti-hypertensive agents therapy such as aldosterone receptor blocker, angiotensin-converting enzyme inhibitors, and calcium channel blockers are the alternative mode of surgical treatment of an aldosteroneproducing adrenal tumor.

\section{REFERENCES}

1. Goto A, Takahashi Y, Kishimoto M, et al, 2009 Primary aldosteronism associated with severe rhabdomyolysis due to profound hypokalemia. Intern Med 48: 219-223.

2. Etgen T, Grabert C, 2009 Tetraparesis with hypertensive crisis: hypokalemic rhabdomyolysis in primary hyperaldosteronism. Nervenarzt 80: 717-719.

3. Kotsaftis P, Savopoulos C, Agapakis D, et al, 2009 Hypokalemia induced myopathy as first manifestation of primary hyperaldosteronism - an elderly patient with unilateral adrenal hyperplasia: a case report. Cases J 2: 6813 .

4. Goto A, Takahashi Y, Kishimoto M, et al, 2009 Primary aldosteronism associated with severe rhabdomyolysis due to profound hypokalemia. Intern Med 48: 219-223.

5. Etgen T, Grabert C, 2009 [Tetraparesis with hypertensive crisis: hypokalemic rhabdomyolysis in primary hyperaldosteronism]. Nervenarzt 80: 717-719. 